\title{
Atomic and Nuclear Physics
}

A VIBGYOR

ISSN: 2631-5017

\section{Modified Atomic Orbital Theory Applied to the Study of High Lying (2pns) ${ }^{1,3} P^{\circ}$ and (2pnd) ${ }^{1,3} P^{\circ}$ Rydberg Series of $\mathrm{B}^{+}$}

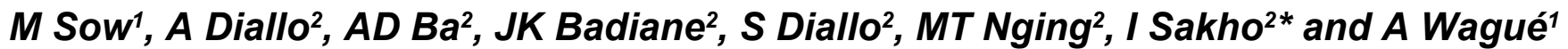

${ }^{1}$ Department of Physics, Atoms Laser Laboratory, Faculty of Sciences and Technologies, University Cheikh Anta Diop, Dakar, Senegal

${ }^{2}$ Department of Physics, UFR Sciences and Technologies, University Assane Seck of Ziguinchor, Ziguinchor, Senegal

\begin{abstract}
We report in this paper energy positions of the $(2 p n s)^{1,3} P^{\circ}$ and $(2 p n d){ }^{1,3} P^{\circ}$ Rydberg states $(n=$ 3-30) and resonance widths of the $(2 p n s){ }^{1} P^{\circ}$ and $(2 p n d){ }^{1} P^{\circ}(n=30)$ members of the $\mathrm{B}^{+}$ion. Calculations are performed in the framework of the Modified Atomic Orbital Theory (MAOT). The present results compared very well to available theoretical and experimental literature values up to $n=24$. The accurate data presented in this work may be a useful guideline for investigators focussing on the photoionization spectrum of the $\mathrm{B}^{+}$ion.

PACS: 31.15.bu - 31.15.vj - 32.80.Zb - 32.80.Ee.

Keywords

Semi-empirical and empirical calculations, Modified Atomic Orbital Theory, Electron correlation calculations for atoms and ions, Autoionizing states, Rydberg series
\end{abstract}

\section{Introduction}

Divalence atomic systems such as the Be isoelectronic sequence are an interesting area for the systematic studies of the photoionization process because of their relatively simple quasi-two-electron structure. Many works have shown that relativistic effects play an important role in the photoionization of small atoms, such as Be [1]. Therefore, it is worthwhile to investigate the interplay between electron-correlation and relativistic effects on the photoionization of atoms or ions with a low nuclear charge. Considerable theoretical and experimental efforts have been made recently to investigate the photoionization of the $\mathrm{B}^{+}$ion. On the experimental side, Janniti, et al. [2] investigated the absorption spectrum of $\mathrm{B}^{+}$for photon energies between 400 and $1700 \AA$ by using two-laser produced plasma; whereas Schippers, et al. [3] studied the photoionization of the $\mathrm{B}^{+} \mathrm{va}-$ lence shell using a photon-ion merged-beams arrangement at the Advanced Light Source (ALS). On the other hand of theoretical side, Tully, et al. [4] reported energy positions for the $2 \mathrm{pns}^{1} \mathrm{P}^{\circ}(\mathrm{n}=4-6)$ and $2 \mathrm{pnd}^{1} \mathrm{P}^{\circ}(\mathrm{n}=4-6)$ levels of $\mathrm{B}^{+}$ along with resonance widths respectively by using R-matrix method; Kim and Manson, [5] investigated the photoionization of the ${ }^{1} S$ ground state of Be-like $\mathrm{B}^{+}$ion leading to the $2 s, 2 p, 3 s$ and $3 p$ states employing a Noniterative Eigenchannel $R$-Matrix (NER-M) method; Hsiao, et al. [6] studied five Rydbergs series of doubly excited $2 p n s^{1,3} P^{\circ}, 2 p$ nd ${ }^{1,3} P^{\circ}$ and $2 p$ nd ${ }^{3} D^{\circ}$ states in the photoionization spectrum of the singly-ionized boron by using the Multiconfiguration Relativistic Random-Phase Approximation (MCRRPA); Sakho, et al. [7] reported accurate results for energy positions of the $(2 p n s)^{1,3} P^{\circ}$ and $(2 p n d)^{1,3} \mathrm{P}^{\circ}$ Rydberg states $(n$ $=3-60)$ along with resonance widths of the $(2 p n s){ }^{1} P^{\circ}$ and $(2 p n d)^{1} P^{\circ}(\mathrm{n}=3-20)$ of the $\mathrm{B}^{+}$ion by using the Screening Constant by Unit Nuclear Charge (SCUNC) method. Using the MAOT method [8-11], Sow, et al. [9] reported accurate energy positions for the $2 \mathrm{p} n \mathrm{~s}^{1,3} \mathrm{P}^{\mathrm{o}}$ and $2 \mathrm{pn} d^{1,3} \mathrm{P}^{\circ}$ levels of the

*Corresponding author: I Sakho, Department of Physics, UFR Sciences and Technologies, University Assane Seck of Ziguinchor, Ziguinchor, Senegal, E-mail: aminafatima_sakho@yahoo.fr

Received: July 17, 2017: Accepted: October 17, 2017: Published: October 19, 2017

Copyright: (c) 2017 Sow M, et al. This is an open-access article distributed under the terms of the Creative Commons Attribution License, which permits unrestricted use, distribution, and reproduction in any medium, provided the original author and source are credited. 
Beryllium atom up to $\mathrm{n}=25$ along with resonance widths in the particular case of the $2 \mathrm{pns}{ }^{1} \mathrm{P}^{\circ}$ states $(\mathrm{n}=3-25)$. In this paper, these previous study are extended to the photoionization spectrum of the Be-like $\mathrm{B}^{+}$ion in the framework of the MAOT formalism. The MAOT-method is known to be a very suitable technique of calculation who has given recently accurate results from simple semi-empirical formulas without needing to compute any photoionization cross section. The purpose of the present work is to report accurate results for energy positions of the (2pns) ${ }^{1,3} \mathrm{P}^{\circ}$ and (2pnd) ${ }^{1,3} \mathrm{P}^{\circ}$ Rydberg states $(\mathrm{n}=3-30)$ along with resonance widths of the (2pns) ${ }^{1} \mathrm{P}^{\circ}$ and (2pnd) ${ }^{1} \mathrm{P}^{\circ}(\mathrm{n}=3-25)$ of the $\mathrm{B}^{+}$ ion in the framework of the MAOT formalism.

\section{Theory}

\section{Brief description of the MAOT formalism}

In the framework of Modified Atomic Orbital Theory (MAOT), total energy of ( $v \ell)$-given orbital is expressed in the form [8-11].

$$
E(v \ell)=-\frac{[Z-\sigma(\ell)]^{2}}{v^{2}}
$$

In Eq. (1), $Z$ stands for the atomic number, $\sigma$ is the screening constant relative to the electron occupying the $v \ell$ orbital, $v$ and $\ell$ denotes respectively the principal quantum number and the orbital quantum number.

For an atomic system of several electrons $\mathrm{M}$, the total energy is given by (in Rydberg)

$$
E=-\sum_{i=1}^{M} \frac{\left[Z-\sigma_{i}(\ell)\right]^{2}}{v_{i}^{2}}
$$

With respect to the usual spectroscopic notation $\left(N \ell, N \ell^{\prime}\right){ }^{2 S+1} L^{\pi}$, this equation becomes

$$
E=-\sum_{i=1}^{M} \frac{\left[Z-\sigma_{i}\left({ }^{2 S+1} L^{\pi}\right)\right]^{2}}{v_{i}^{2}}
$$

$\sigma \mathrm{i}$ denotes the screening constant relative to the i-electron and $v_{\mathrm{i}}$ represents the principal quantum number of the $v_{\mathrm{i}} \ell$ orbital $\left(v_{1}=\mathrm{n}=1\right.$ for $1 \mathrm{~s}, v_{2}=\mathrm{n}=2$ for $2 \mathrm{~s}$ or $2 \mathrm{p}$ and so on). In the photoionisation study, energy resonances are generally measured relatively to the $\mathrm{E}_{\infty}$ converging limit of a given $\left({ }^{2 S+1} \mathrm{~L}_{\mathrm{J}}\right) \mathrm{n} l$-Rydberg series. For these states, the general expression of the energy resonances is given by the formula of Sakho presented previously [11] (in Rydberg units):

$$
E_{n}=E_{\infty}-\frac{1}{n^{2}}\left\{Z-\sigma_{1}\left({ }^{2 S+1} L_{J}\right)-\sigma_{2}\left({ }^{2 S+1} L_{J}\right) \times \frac{1}{n}-\sigma_{2}^{\mu}\left({ }^{2 S+1} L_{J}\right) \times(n-m) \times(n-q) \sum_{k} \frac{1}{f_{k}(n, m, q, s)}\right\}^{2}
$$

In this equation $\mathrm{m}$ and $\mathrm{q}(\mathrm{m}<\mathrm{q})$ denote the principal quantum numbers of the $\left({ }^{2 S+1} \mathrm{~L}_{\mathrm{T}}\right) \mathrm{n} l$-Rydberg series of the considered atomic system used in the empirical determination of the $\sigma_{\mathrm{i}}{ }^{(2 S+1} L_{\mathrm{J}}$ )-screening constants, s represents the spin of the $\mathrm{n} l$-electron $(s=1 / 2), \mathrm{E} \infty$ is the energy value of the series limit generally determined from the NIST atomic database [12], $E_{n}$ denotes the corresponding energy resonance, and $\mathrm{Z}$ represents the nuclear charge of the considered element. The only problem that one may face by using the MAOT formalism is linked to the determination of the $\sum_{k} \frac{1}{f_{k}(n, m, q, s)}$ term. The correct expression of this term is determined iteratively by imposing general Eq. (3) to give accurate data with a constant quantum defect values along all the considered series. The value of $\mu$ is fixed to 1 and 2 during the iteration. The quantum defect $\delta$ is calculated from the standard formula below

$$
E_{n}=E_{\infty}-\frac{R Z_{\text {core }}^{2}}{(n-\delta)^{2}} \Rightarrow \delta=n-Z_{\text {core }} \sqrt{\frac{R}{\left(E_{\infty}-E_{n}\right)}}
$$

\section{Energy of the $2 p n s^{1,3} \mathbf{P}^{\circ}$ and $2 p n d^{1,3} \mathbf{P}^{\circ}$ Rydberg series}

In the framework of the MAOT formalism, the energy positions are given by (in Rydberg units) using Eq. (3)

- For 2 pns ${ }^{1,3} \mathbf{P}^{\circ}$ levels

$$
E_{n}=E_{\infty}-\frac{1}{n^{2}}\left\{Z-\sigma_{1}(s)-\frac{\sigma_{2}(s)}{n}-\sigma_{2}(s) \times(n-m) \times(n-q)\left[\frac{1}{(n+2 m+s)^{3}}+\frac{1}{(n+s)^{4}}-\frac{S}{(n+s)^{2}}-\frac{S}{(n+s)^{3}}\right]\right\}^{2}
$$

- For 2 pnd ${ }^{1,3} \mathbf{P}^{0}$ levels

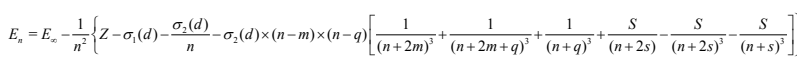

In these equations, $\mathrm{m}$ and $\mathrm{q}(\mathrm{m}<\mathrm{q})$ denote the principal quantum numbers of the 2 pns ${ }^{1} \mathrm{P}^{\circ}$ and 2 pnd ${ }^{1} \mathrm{P}^{\circ}$ levels of $\mathrm{B}^{+}$used in the empirical determination of the $\sigma_{\mathrm{i}}(l=s$ or $d$ ) - screening constants in equations (4) and (5) and $s$ represents the spin of the $n l$-electron $(s=1 / 2) . \mathrm{E}_{\infty}$ is the energy value of the series limit and defined in NIST [12] $\mathrm{E}_{\infty}=31.1533 \mathrm{eV}$. The screening constants in equations (4) and (5) are evaluated using experimental data from Advanced Light Source of Schippers, et al. [3] on $\mathrm{B}^{+}$for the $2 p 4 s^{1} P^{\circ}(\mathrm{m}=4)$ and $2 p 5 s^{1} P^{\circ}(q=5)$ levels respectively equal to (in eV) $26.923 \pm 0.005$ and $28.580 \pm 0.003$ and that of the $2 \mathrm{p}^{3} d^{1} \mathrm{P}^{\circ}(\mathrm{m}=3)$ and $2 \mathrm{p} 4 \mathrm{~d}^{1} \mathrm{P}^{\circ}(\mathrm{q}=4)$ states at $25.458 \pm 0.001$ and $27.889 \pm 0.001$ respectively. The infinite Rydberg $1 \mathrm{Ry}=13.605698 \mathrm{eV}$ is used for energy conversion. Using these experimental data (with $Z=5$ ), we obtain from equations (4) and (5) the empirical values of the screening constants.

$$
\begin{aligned}
& \sigma_{1}(s)=-2.195 \pm 0.004, \sigma_{2}(s)=-4.126 \pm 0.004 . \\
& \sigma_{1}(d)=-2.429 \pm 0.002, \sigma_{2}(d)=0.810 \pm 0.006 .
\end{aligned}
$$

In Eqs. (4) and (5), $S=0$ for the singlet $2 p n s^{1} P^{\circ}$ and $2 p n d^{1} P^{\circ}$ states and $S=1$ for the triplet $2 p n s^{3} P^{\circ}$ and $2 p$ nd ${ }^{3} P^{\circ}$ states.

\section{Natural widths of the $2 p n s{ }^{1} P^{\circ}$ and $2 p n d{ }^{1} P^{\circ}$ series}

The resonance widths of the $2 p n s^{1} P^{\circ}$ and $2 p n d^{1} P^{\circ}$ Rydberg series of the $\mathrm{B}^{+}$ion are given by (in Rydberg units)

\section{- For $2 p n s^{1} P^{\circ}$ levels}

$\Gamma\left(2 p n s ;{ }^{1} P^{0}\right)=\frac{1}{n^{2}}\left\{Z-\sigma_{1}(s)-\frac{\sigma_{2}(s)}{n}+\sigma_{2}(s)(n-m)(n-q)\left[\frac{1}{(n+2 m-q-s)^{3}}-\frac{1}{(n+m-2 s)^{3}}\right]\right\}^{2}$ 
Table 1: Energy positions $(E)$ of doubly $(2 p n s){ }^{1} P^{\circ}$ excited states of $\mathrm{B}^{+}$. The present MAOT results are compared to the Screening Constant by Unit Nuclear Charge (SCUNC) results of Sakho, et al. [7]; Multiconfiguration Relativistic Random-Phase Approximation (MCRRPA) computations of Hsiao, et al. [6]; Noniterative Eigenchannel R-Matrix (NER-M:) results of Kim and Manson [5]; R-Matrix (RM) calculations of Tully, et al. [4]; Advanced Light Source (ALS) experiments of Schippers, et al. [3] and to the Vaccum Ultraviolet (VU) measurements of Janniti, et al. [2]. The results are expressed in eV.

\begin{tabular}{|c|c|c|c|c|c|c|c|}
\hline \multirow[b]{3}{*}{$n s$} & \multicolumn{5}{|l|}{ Theory } & \multicolumn{2}{|c|}{ Experiment } \\
\hline & MAOT & SCUNC & MCRRPA & NER-M & R-M & ALS & VU \\
\hline & \multicolumn{5}{|l|}{$E$} & \multicolumn{2}{|l|}{$E$} \\
\hline $4 s$ & $26.9236(50)$ & $26.9229(50)$ & 26.9137 & 26.9405 & 26.96 & $26.923(5)$ & 26.91 \\
\hline $5 s$ & $28.5804(50)$ & $28.5799(30)$ & 28.5634 & 28.5568 & 28.59 & $28.580(3)$ & 28.58 \\
\hline $6 s$ & $29.4153(20)$ & $29.4201(20)$ & 29.4084 & 29.3924 & 29.42 & $29.420(3)$ & 29.44 \\
\hline $7 s$ & $29.9000(14)$ & $29.9062(14)$ & 29.8983 & 29.8787 & & $29.895(3)$ & \\
\hline $8 s$ & $30.2075(11)$ & $30.2129(11)$ & 30.2075 & 30.1861 & & $30.205(3)$ & \\
\hline $9 s$ & $30.4148(8)$ & $30.4187(8)$ & 30.4151 & 30.3927 & & $30.409(7)$ & \\
\hline $10 \mathrm{~s}$ & $30.5612(7)$ & $30.5636(7)$ & 30.5612 & 30.5382 & & $30.562(9)$ & \\
\hline $11 \mathrm{~s}$ & $30.6683(6)$ & 30.6695 (5) & 30.668 & 30.6444 & & & \\
\hline $12 \mathrm{~s}$ & $30.7490(5)$ & $30.7492(5)$ & 30.7484 & 30.7244 & & & \\
\hline $13 \mathrm{~s}$ & $30.8112(4)$ & 30.8107 (4) & 30.8103 & & & & \\
\hline $14 \mathrm{~s}$ & 30.8602 (3) & 30.8591 (3) & 30.8591 & & & & \\
\hline $15 s$ & $30.8995(3)$ & 30.8979 (3) & 30.8982 & & & & \\
\hline $16 s$ & $30.9314(2)$ & 30.9296 (3) & 30.9301 & & & & \\
\hline $17 s$ & 30.9577 (2) & $30.9557(2)$ & 30.9564 & & & & \\
\hline $18 \mathrm{~s}$ & $30.9796(2)$ & $30.9774(2)$ & 30.9783 & & & & \\
\hline $19 \mathrm{~s}$ & $30.9980(2)$ & $30.9958(2)$ & 30.9968 & & & & \\
\hline $20 \mathrm{~s}$ & $31.0137(2)$ & $31.0114(2)$ & 31.0125 & & & & \\
\hline $21 \mathrm{~s}$ & 31.0271 (1) & 31.0249 (1) & & & & & \\
\hline $22 s$ & 31.0387 (1) & 31.0364 (1) & & & & & \\
\hline $23 \mathrm{~s}$ & 31.0487 (1) & 31.0465 (1) & & & & & \\
\hline $24 s$ & 31.0575 (1) & 31.0554 (1) & & & & & \\
\hline $25 s$ & 31.0652 (1) & & & & & & \\
\hline $26 s$ & 31.0721 (1) & & & & & & \\
\hline $27 s$ & $31.0782(1)$ & & & & & & \\
\hline $28 \mathrm{~s}$ & 31.0836 (1) & & & & & & \\
\hline $29 \mathrm{~s}$ & 31.0884 (1) & & & & & & \\
\hline $30 \mathrm{~s}$ & $31.0928(1)$ & & & & & & \\
\hline$\ldots$ & & & & & & & \\
\hline$\infty s$ & 31.1533 & & & & & & \\
\hline
\end{tabular}

\section{- For $2 p n d^{1} P^{\circ}$ levels}

$\Gamma\left(2 p n d ; P^{0}\right)=\frac{1}{n^{2}}\left\{Z-\sigma_{1}(d)-\frac{\sigma_{2}(d)}{n}-\sigma_{2}(d)(n-m)(n-q)\left[\frac{1}{(n+5 m)^{3}}+\frac{1}{(n+2 m-q)^{3}}+\frac{1}{(n-m+s)^{3}}\right]\right\}$

The screening constants in equations (6) and (7) are evaluated using a photon-ion merged-beams arrangement results [3] on $\mathrm{B}^{+}$for the $2 \mathrm{p} 4 \mathrm{~s}^{1} \mathrm{P}^{\circ}(\mathrm{m}=4)$ and $2 \mathrm{p} 5 \mathrm{~s}$ ${ }^{1} \mathrm{P}^{\circ}(\mathrm{q}=5)$ levels respectively equal to (in $\left.\mathrm{eV}\right) 0.220 \pm$ 0.001 and $0.106 \pm 0.007$ and that of the $2 p 3 d^{1} P^{\circ}(\mathrm{m}=3)$ and $2 p 4 d^{1} \mathrm{P}^{\circ}(\mathrm{q}=4)$ states at $0.034 \pm 0.002$ and $0.016 \pm$ 0.002 respectively. Using these experimental data (with $\mathrm{Z}$ $=5$ ), we obtain from equations (6) and (7) the empirical values of the screening constants

$$
\begin{aligned}
& \sigma_{1}(\mathrm{~s})=4.3652 \pm 0.0001, \sigma_{2}(\mathrm{~s})=-4.9656 \pm 0.0001, \\
& \sigma_{1}(\mathrm{~d})=4.6357 \pm 0.0001, \sigma_{2}(\mathrm{~d})=0.5665 \pm 0.0001 .
\end{aligned}
$$

\section{Results and Discussions}

The results of the presently calculations for the energy positions of doubly $(2 p n s)^{1,3} P^{\circ}$ and $(2 p n d)^{1,3} P^{\circ}$ excited state of the $\mathrm{B}^{+}$atom are listed in Table 1, Table 2, Table 3 ,
Table 4 and Table 5. In Table 1 we present a comparison of energy positions of the doubly $2 \mathrm{pns}{ }^{1} \mathrm{P}^{\circ}$ excited states of $\mathrm{B}^{+}$atom obtained from the present Modified Atomic Orbital Theory (MAOT) with the absorption spectrum of B II in the vacuum ultraviolet results of Janniti, et al. [2], the R-Matrix (R-M) calculations of Tully, et al. [4], the Noniterative Eigenchannel R-Matrix (NER-M) results Kim and Manson [5], the Multiconfiguration Relativistic Random-Phase Approximation (MCRRPA) computations of Hsiao, et al. [6], the Screening Constant by Unit Nuclear Charge SCUNC values of Sakho, et al. [7], and with the Advanced Light Source (ALS) experiments of Schippers, et al. [3]. On the other hand, our present results are quoted with uncertainties. It can be seen that the present MAOT results agrees very well with the cited literature data. It should be mentioned the good agreements between the calculations for the $2 p 10 s{ }^{1} P^{\circ}$ level, and the results for which the principal quantum number range between 12 and 20. Specially, for the $2 p 20 s^{1} P^{\circ}$ level, the present MAOT data at $31.0137 \mathrm{eV}$ agrees very 
Table 2: Energy positions $(E)$ of doubly $(2 p n d){ }^{1} P^{\circ}$ excited states of $\mathrm{B}^{+}$. The present MAOT results are compared to the Screening Constant by Unit Nuclear Charge (SCUNC) results of Sakho, et al. [7]; Multiconfiguration Relativistic Random-Phase Approximation (MCRRPA) computations of Hsiao, et al. [6]; Noniterative Eigenchannel R-Matrix (NER-M:) results of Kim and Manson [5]; R-Matrix (RM) calculations of Tully, et al. [4]; Advanced Light Source (ALS) experiments of Schippers, et al. [3] and to the Vaccum Ultraviolet (VU) measurements of Janniti, et al. [2]. The results are expressed in eV.

\begin{tabular}{|c|c|c|c|c|c|c|c|}
\hline \multirow[b]{3}{*}{ nd } & \multicolumn{5}{|l|}{ Theory } & \multicolumn{2}{|c|}{ Experiment } \\
\hline & MAOT & SCUNC & MCRRPA & NER-M & R-M & ALS & VU \\
\hline & \multicolumn{5}{|l|}{$E$} & \multicolumn{2}{|l|}{$E$} \\
\hline $3 d$ & $25.4587(10)$ & $25.4578(10)$ & 25.5731 & 25.4262 & 25.47 & $25.458(1)$ & 25.43 \\
\hline $4 d$ & $27.8894(10)$ & $27.8889(10)$ & 27.9453 & 27.861 & 27.90 & $27.889(1)$ & 27.91 \\
\hline $5 d$ & $29.0432(8)$ & $29.0443(8)$ & 29.0747 & 29.0166 & 29.05 & 29.041 (1) & 29.06 \\
\hline $6 d$ & $29.6790(6)$ & $29.6804(6)$ & 29.6983 & 29.6533 & 29.68 & $29.676(1)$ & 29.7 \\
\hline $7 d$ & $30.0659(5)$ & $30.0670(5)$ & 30.0784 & 30.0408 & & $30.064(2)$ & \\
\hline $8 d$ & $30.3184(4)$ & $30.3194(4)$ & 30.3269 & 30.2935 & & $30.320(2)$ & \\
\hline $9 d$ & $30.4923(3)$ & 30.4931 (3) & 30.4983 & 30.4676 & & $30.490(4)$ & \\
\hline $10 d$ & $30.6170(3)$ & 30.6177 (3) & 30.6215 & 30.5924 & & $30.610(1)$ & \\
\hline $11 d$ & $30.7095(2)$ & $30.7101(2)$ & 30.713 & 30.685 & & & \\
\hline $12 d$ & $30.7800(2)$ & $30.7805(2)$ & 30.7828 & 30.7556 & & & \\
\hline $13 d$ & $30.8349(2)$ & $30.8354(2)$ & 30.8372 & & & & \\
\hline $14 d$ & $30.8785(1)$ & $30.8790(1)$ & 30.8805 & & & & \\
\hline $15 d$ & $30.9138(1)$ & $30.9142(1)$ & 30.9156 & & & & \\
\hline $16 d$ & $30.9426(1)$ & $30.9430(1)$ & 30.9443 & & & & \\
\hline $17 d$ & 30.9666 (1) & 30.9669 (1) & 30.9681 & & & & \\
\hline $18 d$ & 30.9867 (1) & $30.9870(1)$ & 30.9881 & & & & \\
\hline $19 d$ & $31.0036(1)$ & $31.0040(1)$ & 31.0051 & & & & \\
\hline $20 d$ & $31.0182(1)$ & 31.0185 (1) & 31.0196 & & & & \\
\hline $21 d$ & 31.0307 (1) & $31.0310(1)$ & & & & & \\
\hline $22 d$ & 31.0415 (1) & $31.0418(1)$ & & & & & \\
\hline $23 d$ & $31.0510(1)$ & $31.0513(1)$ & & & & & \\
\hline $24 d$ & 31.0593 (1) & 31.0596 (1) & & & & & \\
\hline $25 d$ & 31.0666 (1) & & & & & & \\
\hline $26 d$ & 31.0731 (1) & & & & & & \\
\hline $27 d$ & 31.0789 (1) & & & & & & \\
\hline $28 d$ & 31.0841 (1) & & & & & & \\
\hline $29 d$ & 31.0888 (1) & & & & & & \\
\hline $30 d$ & 31.0930 (1) & & & & & & \\
\hline \multicolumn{8}{|l|}{$\ldots$} \\
\hline$\infty d$ & 31.1533 & & & & & & \\
\hline
\end{tabular}

satisfactory with the SCUNC [7] and the MCRRPA [6] calculations respectively at $31.0114 \mathrm{eV}$ and $31.0125 \mathrm{eV}$. Here the present deviations with respect to the SCUNC [7] and the MCRRPA [6] calculations respectively equal to at $0.0023 \mathrm{eV}$ and $0.0012 \mathrm{eV}$. For the $2 p 24 s^{1} \mathrm{P}^{\circ}$ level, the present MAOT equal to $31.0575 \mathrm{eV}$ is shifted at 0.0021 $\mathrm{eV}$ to the $31.0554 \mathrm{eV} \mathrm{SCUNC} \mathrm{[7]} \mathrm{prediction.} \mathrm{These} \mathrm{good}$ agreements enable one to expect as accurate the present MAOT calculations for high lying $2 \mathrm{pns}^{1} \mathrm{P}^{\circ}(\mathrm{n} \geq 20)$ levels. Table 2 indicates the present MAOT calculations for the 2pnd ${ }^{1} \mathrm{P}^{\circ}$ compared to the experimental measurements $[2,3]$ and theoretical calculations [4-7]. Here again, the agreements between the current results and the quoted literature data are seen to be very good. Thus up to $2 p 12 d$ ${ }^{1} P^{\circ}$ level, the agreements between theory and experiment is seen to be very good. For the high lying level $2 p 20 d^{1} P^{\circ}$ the agreement the present MAOT data at $31.01818 \mathrm{eV}$ agrees well with the SCUNC [7] and the MCRRPA [6] calculations respectively at $31.0185 \mathrm{eV}$ and $31.0196 \mathrm{eV}$. Besides, it should be mentioned the very good agreement between the present MAOT and the SCUNC [7] predictions up to $2 p 20 d^{1} P^{\circ}$. As a result, our listed data for $\mathrm{n}$ $>25$ are expected to be accurate. Table 3 indicates the present MAOT calculations for the $2 p n s{ }^{3} P^{\circ}$ and $2 p n d$ ${ }^{3} P^{\circ}$ doubly excited states compared to the SCUNC results [7] and to the MCRRPA values [6]. For both $2 p n s^{3} P^{\circ}$ and $2 p n d^{3} P^{\circ}$ states up to $\mathrm{n}=20$, the agreements between the calculations are seen to be very good. These agreements allow us to extend the MAOT calculations up to $n=30$ and the data are expect them to be accurate. As far as the natural widths of the $2 \mathrm{pns}{ }^{1} \mathrm{P}^{\circ}$ and $2 \mathrm{pns}{ }^{1} \mathrm{P}^{\circ}$ Rydberg states are concerned, the results obtained in this work using Eq. (5) and (6) are respectively listed in Table 4 and Table 5 an converted into $(\mathrm{eV})$ for direct comparison with the ALS experimental values of Schippers, et al. [3] and with the theoretical R-M calculations of Tully, et al. 
Table 3: Energy $(E)$ of doubly $(2 p n s){ }^{3} P^{\circ}$ and $(2 p n d){ }^{3} P^{\circ}$ excited states of $\mathrm{B}^{+}$. The present MAOT results are compared to the Screening Constant by Unit Nuclear Charge (SCUNC) results of Sakho, et al. [7] and to the Multiconfiguration Relativistic Random-Phase Approximation (MCRRPA) computations of Hsiao, et al. [6]. The results are expressed in eV.

\begin{tabular}{|c|c|c|c|c|c|c|c|}
\hline & MAOT & SCUNC & MCRRPA & & MAOT & SCUNC & MCRRPA \\
\hline \multicolumn{4}{|c|}{ ns } & \multicolumn{4}{|c|}{$n d$} \\
\hline & & & & $3 d$ & $25.1527(20)$ & $25.1515(32)$ & 25.1505 \\
\hline $4 s$ & $26.6636(50)$ & $26.7126(53)$ & 26.705 & $4 d$ & $27.7556(15)$ & 27.7757 (18) & 27.7498 \\
\hline $5 s$ & 28.4759 (33) & $28.4836(32)$ & 28.4707 & $5 d$ & $28.9720(15)$ & $28.9891(12)$ & 28.9706 \\
\hline $6 s$ & $29.3654(30)$ & $29.3673(22)$ & 29.3586 & $6 d$ & $29.6364(10)$ & $29.6490(8)$ & 29.637 \\
\hline $7 s$ & $29.8733(20)$ & $29.8739(16)$ & 29.868 & $7 d$ & $30.0383(10)$ & $30.0472(6)$ & 30.0394 \\
\hline $8 s$ & $30.1919(20)$ & $30.1915(12)$ & 30.1874 & $8 d$ & $30.2995(10)$ & 30.3060 (5) & 30.3008 \\
\hline 9s & $30.4051(2)$ & $30.4038(9)$ & 30.4008 & $9 d$ & $30.4788(10)$ & $30.4835(4)$ & 30.4801 \\
\hline $10 \mathrm{~s}$ & $30.5548(2)$ & $30.5528(7)$ & 30.5504 & $10 d$ & $30.6070(10)$ & $30.6106(3)$ & 30.6083 \\
\hline $11 \mathrm{~s}$ & $30.6640(2)$ & $30.6613(6)$ & 30.6593 & 11d & $30.7019(10)$ & 30.7046 (3) & 30.7032 \\
\hline $12 \mathrm{~s}$ & $30.7460(2)$ & $30.7429(5)$ & 30.741 & $12 d$ & $30.7741(10)$ & $30.7762(2)$ & 30.7754 \\
\hline $13 \mathrm{~s}$ & $30.8090(2)$ & 30.8057 (4) & 30.8039 & $13 d$ & $30.8302(10)$ & $30.8319(2)$ & 30.8315 \\
\hline $14 s$ & $30.8586(2)$ & 30.8551 (3) & 30.8533 & $14 d$ & 30.8747 (10) & $30.8761(2)$ & 30.8761 \\
\hline $15 \mathrm{~s}$ & $30.8983(2)$ & 30.8947 (3) & 30.8929 & $15 d$ & 30.9107 (10) & $30.9118(2)$ & 30.912 \\
\hline $16 s$ & $30.9304(2)$ & 30.9269 (3) & 30.925 & $16 d$ & $30.9400(7)$ & 30.9410 (1) & 30.9414 \\
\hline $17 s$ & $30.9569(2)$ & $30.9534(2)$ & 30.9515 & $17 d$ & $30.9644(7)$ & 30.9652 (1) & 30.9658 \\
\hline $18 \mathrm{~s}$ & $30.9790(2)$ & $30.9755(2)$ & 30.9736 & $18 d$ & $30.9848(7)$ & 30.9855 (1) & 30.9862 \\
\hline $19 \mathrm{~s}$ & $30.9975(2)$ & $30.9942(2)$ & 30.9922 & $19 d$ & $31.0021(5)$ & 31.0027 (1) & 31.0035 \\
\hline $20 \mathrm{~s}$ & $31.0133(2)$ & $31.0100(2)$ & 31.008 & $20 d$ & $31.0168(5)$ & $31.0174(1)$ & 31.0183 \\
\hline $21 \mathrm{~s}$ & $31.0268(2)$ & 31.0236 (1) & & 21d & $31.0295(2)$ & 31.0300 (1) & \\
\hline $22 s$ & $31.0384(2)$ & 31.0354 (1) & & $22 d$ & $31.0405(2)$ & & \\
\hline $23 s$ & $31.0485(2)$ & & & $23 d$ & $31.0501(2)$ & & \\
\hline $24 s$ & $31.0573(2)$ & & & $24 d$ & $31.0585(2)$ & & \\
\hline $25 \mathrm{~s}$ & $31.0651(2)$ & & & $25 d$ & $31.0659(2)$ & & \\
\hline $26 s$ & $31.0719(1)$ & & & $26 d$ & $31.0725(2)$ & & \\
\hline $27 s$ & 31.0780 (1) & & & $27 d$ & $31.0784(1)$ & & \\
\hline $28 s$ & 31.0835 (1) & & & $28 d$ & $31.0836(1)$ & & \\
\hline $29 \mathrm{~s}$ & $31.0883(1)$ & & & $29 d$ & $31.0883(1)$ & & \\
\hline $30 \mathrm{~s}$ & 31.0927 (1) & & & $30 d$ & 31.0926 (1) & & \\
\hline$\ldots$ & $\ldots$ & $\ldots$ & $\ldots$ & $\ldots$ & & & \\
\hline$\infty s$ & 31.1533 & & & $\ldots$ & & & \\
\hline
\end{tabular}

Table 4: Resonance widths $\left(\Pi\right.$ ) of doubly $(2 p n s){ }^{1} P^{\circ}$ excited states of $\mathrm{B}^{+}$. The present MAOT results are compared to the Screening Constant by Unit Nuclear Charge (SCUNC) results of Sakho, et al. [7]; Multiconfiguration Relativistic Random-Phase Approximation (MCRRPA) computations of Hsiao, et al. [6]; Noniterative Eigenchannel R-Matrix (NER-M:) results of Kim and Manson [5]; R-Matrix (R-M) calculations of Tully, et al. [4] and to the Advanced Light Source (ALS) experiments of Schippers, et al. [3] The a (-b) (c) notation means $a \times 10^{-b}$ and $(c)$ indicate the uncertainties of the experimental widths given in parentheses. The results are expressed in eV.

\begin{tabular}{|c|c|c|c|c|c|c|}
\hline & & Theory & & & & Experiment \\
\hline States & MAOT & SCUNC & MCRRPA & NER-M & R-M & ALS \\
\hline$(2 p 4 s)^{1} P^{\circ}$ & $2.220(-1)(80)$ & $2.212(-1)$ & $2.663(-1)$ & $2.453(-1)$ & $2.41(-1)$ & $2.20(-1)(1)$ \\
\hline$(2 p 5 s)^{1} P^{\circ}$ & $1.060(-1)(70)$ & $1.066(-1)$ & $1.188(-1)$ & $1.153(-1)$ & $1.10(-1)$ & $1.06(-1)(7)$ \\
\hline$(2 p 6 s)^{1} P^{\circ}$ & $5.920(-2)(60)$ & $5.969(-2)$ & $6.343(-2)$ & $6.350(-2)$ & $5.90(-2)$ & $4.80(-2)(6)$ \\
\hline$(2 p 7 s)^{1} P^{\circ}$ & $3.660(-2)(60)$ & $3.685(-2)$ & $3.788(-2)$ & $3.840(-2)$ & & $2.90(-2)(7)$ \\
\hline$(2 p 8 s)^{1} P^{\circ}$ & $2.437(-2)(50)$ & $2.437(-2)$ & $2.442(-2)$ & $2.490(-2)$ & & $2.00(-2)(6)$ \\
\hline$(2 p 9 s)^{1} P^{\circ}$ & $1.715(-2)(50)$ & $1.696(-2)$ & $1.663(-2)$ & $1.710(-2)$ & & $4.00(-2)(1)$ \\
\hline$(2 p 10 s)^{1} P^{\circ}$ & $1.261(-2)(30)$ & $1.230(-2)$ & $1.179(-2)$ & $1.220(-2)$ & & $2.00(-2)(2)$ \\
\hline$(2 p 11 s)^{1} P^{\circ}$ & $9.593(-3)(30)$ & $9.207(-3)$ & $8.629(-3)$ & $9.000(-3)$ & & \\
\hline$(2 p 12 s)^{1} P^{\circ}$ & $7.507(-3)(30)$ & $7.081(-3)$ & $6.465(-3)$ & $6.900(-3)$ & & \\
\hline
\end{tabular}

[4], NER-M results of Kim and Manson [5], MCRRPA values of Hsiao, et al. [6], and with the SCUNC results of Sakho, et al. [7]. Here, the agreements between the current MOAT results and all the listed theoretical results are seen to be good up to $n=12$. Overall, although good agreements are obtained between the present MAOT calculations and the quoted literature data, it should mentioned slight discrepancies between the present results and those from the NER-M computations [5]. Let us for instance consider the 10s-state for which ab initio 
Table 5: Resonance widths $\left(\Pi\right.$ ) of doubly $(2 p n d){ }^{1} P^{\circ}$ excited states of $\mathrm{B}^{+}$. The present MAOT results are compared to the Screening Constant by Unit Nuclear Charge (SCUNC) results of Sakho, et al. [7]; Multiconfiguration Relativistic Random-Phase Approximation (MCRRPA) computations of Hsiao, et al. [6]; Noniterative Eigenchannel R-Matrix (NER-M:) results of Kim and Manson [5]; R-matrix (R-M) calculations of Tully, et al. [4] and to the Advanced Light Source (ALS) experiments of Schippers, et al. [3]. The a (-b) (c) notation means $a \times 10^{-b}$ and $(c)$ indicates the uncertainties of the experimental widths given in parentheses. The results are expressed in eV.

\begin{tabular}{|l|l|l|l|l|l|l|}
\hline \multicolumn{3}{|c|}{ Theory } & \multicolumn{3}{l|}{ Experiment } \\
\hline States & MAOT & SCUNC & MCRRPA & NER-M & R-M & ALS \\
\hline$(2 p 3 d)^{1} P^{\circ}$ & $3.40(-2)(20)$ & $3.40(-2)$ & $5.17(-2)$ & $3.12(-2)$ & $3.38(-2)$ & $3.4(-2)(2)$ \\
\hline$(2 p 4 d)^{1} P^{\circ}$ & $1.60(-2)(20)$ & $1.59(-2)$ & $2.21(-2)$ & $1.41(-2)$ & $1.78(-2)$ & $1.6(-2)(2)$ \\
\hline$(2 p 5 d)^{1} P^{\circ}$ & $1.04(-2)(12)$ & $9.28(-3)$ & $1.14(-2)$ & $9.5(-3)$ & $5.90(-2)$ & $1.0(-2)(3)$ \\
$(2 p 6 d)^{1} P^{\circ}$ & $6.63(-3)(10)$ & $6.07(-3)$ & $6.61(-3)$ & $6.4(-3)$ & $2.41(-1)$ & $8.0(-3)(3)$ \\
\hline$(2 p 7 d)^{1} P^{\circ}$ & $4.54(-3)(8)$ & $4.29(-3)$ & $4.17(-3)$ & $4.4(-3)$ & & $8.0(-3)(4)$ \\
\hline$(2 p 8 d)^{1} P^{\circ}$ & $3.30(-3)(7)$ & $3.11(-3)$ & $2.79(-3)$ & $3.1(-3)$ & & $1.0(-3)(6)$ \\
\hline$(2 p 9 d)^{1} P^{\circ}$ & $2.51(-3)(6)$ & $2.44(-3)$ & $1.96(-3)$ & $2.3(-3)$ & & $5.0(-3)(8)$ \\
\hline
\end{tabular}

and experimental data are available. The present MOAT prediction at $30.5612 \mathrm{eV}$ is to be compared to the Noniterative Eigenchannel R-Matrix (NER-M) result of Kim and Manson [5] at $30.5382 \mathrm{eV}$, to the Multiconfiguration Relativistic Random-Phase Approximation (MCRRPA) data of Hsiao, et al. [6] at $30.5612 \mathrm{eV}$ and to the Advanced Light Source (ALS) experiments of Schippers, et al. [3] equals to 30.562 (9) eV. The energy deviations between the MAOT data and the NER-M, MCRRPA and the ALS data are respectively equal to $0.023 \mathrm{eV}, 0.000$ $\mathrm{eV}$ and $0.0008 \mathrm{eV}$. This indicates that the NER-M computation is less accurate than both the MCRRPA calculation ALS measurement in contrast with the MAOT prediction agreeing excellently with both the MCRRPA and ALS data. In addition, for the high 12s-state, the MAOT, NER-M and MCRRPA results are respectively at $30.74905 \mathrm{eV}, 30.7244 \mathrm{eV}$ and $30.7484 \mathrm{eV}$. For this level, the energy deviations between the MAOT prediction and the NER-M and MCRRPA computations are respectively at $0.02465 \mathrm{eV}$ and $0.00065 \mathrm{eV}$. Here again, the MAOT forecast matches more with the MCRRPA data. The same discrepancies are observed in Table 2 for the nd levels. For the 10d-state, the MAOT, NER-M, MCRRPA and ALS data are respectively at $30.6170 \mathrm{eV}$, $30.5924 \mathrm{eV}, 30.6215 \mathrm{eV}$, and 30.610 (1) eV. The energy deviations between the MAOT calculation and the cited literature data are respectively equal to $0.0246 \mathrm{eV}$, $0.0042 \mathrm{eV}$ and $0.007 \mathrm{eV}$. For the 12d-level, the MAOT, NER-M and MCRRPA results are respectively at 30.7800 $\mathrm{eV}, 30.7556 \mathrm{eV}$ and $30.7828 \mathrm{eV}$. The corresponding energy deviations between the MAOT prediction and the NER-M and MCRRPA computations are respectively at $0.0244 \mathrm{eV}$ and $0.0028 \mathrm{eV}$. Here again, the MAOT forecast matches more with the ALS and the MCRRPA data for the 10d-level and with the MCRRPA result for the $12 \mathrm{~d}$ level. As explained by Hsiao, et al. [6], in the MCRR$\mathrm{PA}$ formalism relativistic and correlation effects are included in the calculation done in the framework of the $j j$ coupling. The discrepancies between the MAOT calculations and the NER-M computations may be explained arguing that relativistic and correlation effects are prob- ably not well taken into account in the NER-M formalism [5] combined with multichannel quantum-defect theory at the $R$-matrix surface. In the MAOT formalism, the $\sigma_{\mathrm{i}}$-screening constants are evaluated using experimental data incorporating all relativistic and electron correlation effects. The excellent agreements between the MAOT calculations and both the MCRRPA [6] computations and the ALS [3] measurements indicate that relativistic and correlation effects are well incorporated in the $\sigma_{\mathrm{i}}$-screening constants up to high $n=30$ levels.

\section{Conclusion}

The energy positions of the $2 p n s^{1,3} P^{\circ}$ and $2 p n d^{1,3} P^{\circ}$ Rydberg series and widths of the $(2 p n s)^{1} P^{\circ}$ and $(2 p n d)^{1} P^{\circ}$ excited states of the $\mathrm{B}^{+}$ion are presented in this paper using the Modified Atomic Orbital Theory (MAOT). In general, the present results agree very well with both quoted theoretical and experimental literature data. For $n \geq 25$, no theoretical and experimental literature values are available for direct comparison. It is shown through this study the simplicity of the formalism in contrast with the ab initio methods cited in the paper. In addition, although the energy resonances of the (2pns) $)^{1,3} \mathrm{P}^{\circ}$ and $(2 p n d)^{1,3} \mathrm{P}^{\circ}$ Rydberg states members of the $\mathrm{B}^{+}$ion have been widely calculated by many ab initio methods, the present MAOT calculations have been extended to the high-n Rydberg states and precise data are tabulated within simple analytical formulas up to $n=30$. The good accuracy obtained in this work point out that, the Modified Atomic Orbital Theory is suitable for the interpretation of atomic spectra. Extension of the present MAOT formalism to the Be-like $(Z>5)$ ions is then very challenging.

\section{References}

1. Yoshida F, Matsuoka L, Osaki H, Kikkawa S, Azuma Y, et al. (2006) Observation of perturbed 3snp double photoexcited Rydberg series of beryllium atoms. Phys Rev A 73.

2. Janniti E, Pinzhong F, Tondello G (1986) The absorption spectrum of $b$ ii in the vacuum ultraviolet. Phys Scr 33: 434-441.

3. Schippers S, Müller A, McLaughlin BM, Aguilar A, Cisneros C, et al. (2003) Photoionization studies of the $B+$ valence shell: Experiment and theory. J Phys B At Mol Opt Phys 36: 33713381. 
4. Tully JA, Dourneuf ML, Zeippen CJ (1989) Photoionisation of the $\mathrm{B}+(1 \mathrm{Se})$ ground state. Astronomy and Astrophysics 211: 485-494.

5. Kim DS, Manson ST (2004) Photoionization of the groundstate Be-like $B+$ ion leading to the $n=2$ and $n=3$ states of B2+. J Phys B At Mol Opt Phys 37: 4013.

6. Hsiao JT, Shiao HT, Huang KN (2009) Doubly-excited triplet Rydberg series of the B+ ion. Chin J Phys 47: 173.

7. Sakho I, Diop B, Faye M, Sène A, Guèye, et al. (2013) Screening constant by unit nuclear charge calculations of high lying (2pns) $1,3 \mathrm{P}^{\circ}$ and (2pnd) $1,3 \mathrm{P}^{\circ}$ states of the $\mathrm{B}+$ ion. Atom Data Nucl Data Tabl 99: 447-458.

8. Sakho I (2013) Photoabsorption of $\mathrm{H}$ - and $\mathrm{He}$ via the modified atomic orbital theory: Application to the $1 \mathrm{P}^{\circ}$ - rydberg states. Chin J Phys 51: 209.
9. Sow M, Dieng M, Tine M, Faye M, Diop B, et al. (2014) Calculations of high lying (2pns) $1,3 \mathrm{P}^{\circ}$ and (2pnd) $1,3 \mathrm{P}^{\circ}$ Rydberg states of $\mathrm{Be}$ atom via the modified atomic orbital theory. Chin J Phys 52: 1459.

10. Sakho I (2014) General formalism of the modified atomic orbital theory for the Rydberg series of atoms and ions: Application to the photoionization of $\mathrm{Ne}^{+}$. J At Mol Sci 5: 206-216.

11. Diop B, Faye M, Dieng M, Sow M, Guèye M, et al. (2014) Modified Orbital Atomic Study of Dominant Rydberg series in the Photoionization Spectra of Halogen-like $\mathrm{Kr}+$ and $\mathrm{Xe}+$ ions. Chin J Phys 52: 1227-1237.

12. Martin MC (1999) NIST Atomic Spectra Data Base. (2nd edn), National Institute of Standards and Technology Gaithersburg, Maryland, USA. 